\title{
Re-examination of the Controversial Coexistence of Traumatic Brain Injury and Posttraumatic Stress Disorder: Misdiagnosis and Self-Report Measures
}

\author{
Robert J. Sbordone • Ronald M. Ruff
}

Received: 15 September 2009 / Accepted: 10 January 2010 / Published online: 16 March 2010

(C) The Author(s) 2010. This article is published with open access at Springerlink.com

\begin{abstract}
The coexistence of traumatic brain injury (TBI) and posttraumatic stress disorder (PTSD) remains a controversial issue in the literature. To address this controversy, we focused primarily on the civilian-related literature of TBI and PTSD. Some investigators have argued that individuals who had been rendered unconscious or suffered amnesia due to a TBI are unable to develop PTSD because they would be unable to consciously experience the symptoms of fear, helplessness, and horror associated with the development of PTSD. Other investigators have reported that individuals who sustain TBI, regardless of its severity, can develop PTSD even in the context of prolonged unconsciousness. A careful review of the methodologies employed in these studies reveals that investigators who relied on clinical interviews of TBI patients to diagnose PTSD found little or no evidence of PTSD. In contrast, investigators who relied on PTSD questionnaires to diagnose PTSD found considerable evidence of PTSD. Further analysis revealed that many of the TBI patients who were initially diagnosed with PTSD according to self-report questionnaires did not meet the diagnostic criteria for PTSD upon completion of a clinical interview. In particular, patients with severe TBI were often misdiagnosed with PTSD. A number of investigators found
\end{abstract}

Copies of this paper can be obtained from Robert J. Sbordone.

R. J. Sbordone $(\bowtie)$

24422 Avenida de la Carlota, Suite 270,

Laguna Hills, CA 92653, USA

e-mail: bobneurpsy@aol.com

R. M. Ruff

San Francisco Clinical Neurosciences,

909 Hyde Street,

San Francisco, CA 94109, USA

e-mail: ronruff@mindspring.com that many of the severe TBI patients failed to follow the questionnaire instructions and erroneously endorsed PTSD symptoms because of their cognitive difficulties. Because PTSD questionnaires are not designed to discriminate between PTSD and TBI symptoms or determine whether a patient's responses are accurate or exaggerated, studies that rely on self-report questionnaires to evaluate PTSD in TBI patients are at risk of misdiagnosing PTSD. Further research should evaluate the degree to which misdiagnosis of PTSD occurs in individuals who have sustained mild TBI.

Keywords Posttraumatic stress disorder .

Traumatic brain injury

\section{Introduction}

There has been a great deal of controversy in the literature about whether the diagnosis of traumatic brain injury (TBI) and posttraumatic stress disorder (PTSD) can coexist. On one hand, most clinicians agree that PTSD and TBI can coexist for individuals who consciously experience a lifethreatening injury before or after they sustain a brain injury. For example, if a woman is sexually assaulted and subsequently struck on the head repeatedly until she is rendered unconscious, she is likely to have PTSD and TBI especially in the absence of retrograde amnesia. However, a dispute emerges as to whether individuals can develop PTSD if the TBI resulted in a loss of consciousness and retrograde and/or anterograde amnesia for the traumatic events. Some investigators (e.g., Sbordone and Liter 1995) have argued that these individuals are unable to develop PTSD because they will not consciously experience feelings of fear, helplessness, and horror. Other investigators have reported that individuals with TBI can develop PTSD 
while being unconscious for extended periods of time (e.g., Bryant 2001). To examine this controversy, in this paper, first, we provide a brief historical background of PTSD, followed by the current definitions for acute stress disorder (ASD) and PTSD. Second, we describe the diagnostic criteria for TBI according to severity. In a third section, we discuss the overlap that exists between PTSD and mild TBI patients with postconcussive symptoms. In the fourth section, we address the following questions: (a) Does a TBI prevent the development of PTSD? (b) Does TBI increase the risk for PTSD? (c) How do TBI patients develop PTSD? (d) Do PTSD patients have cognitive deficits? (e) Are the cognitive deficits of PTSD patients due to PTSD-induced brain changes? (f) Can describing the traumatic event during a clinical interview and/or being administered PTSD questionnaires negatively affect a PTSD patient's performance on neuropsychological tests?

\section{Historical Background of PTSD}

PTSD gained acceptance in the medical and psychiatric community after a number of studies appeared in prominent medical and psychiatric journals describing the psychiatric symptoms of soldiers who had served and fought in the Vietnam War (Fox 1972; Goldsmith and Cretekos 1969). Subsequent publications described the profound psychological and emotional sequelae secondary to being exposed to extremely traumatic events such as fires, explosions, floods, torture, serious motor vehicle accidents (MVAs), violent crimes, and sexual assault (e.g., Horowitz 1976). These publications posited that individuals with PTSD no longer felt in control of their lives, viewed the world as an unpredictable and dangerous place, lived in fear, and reported a loss of trust in a "just world." These studies also reported that individuals with PTSD avoided thoughts or situations that might trigger traumatic memories, complained of feelings of emotional numbness, and exhibited symptoms of blunted affect.

The conceptual refinements coupled with the empirical information generated by these studies significantly advanced our understanding of individuals who have been exposed to a traumatic stressor. This led in 1980 to the inclusion of the diagnostic criteria for PTSD in the Diagnostic and Statistical Manual of Mental Disorders III (DSM-III; American Psychiatric Association 1980). At that time, PTSD was defined as a syndrome that developed in response to a "stressor that would evoke significant symptoms of distress in almost everyone" (p. 238). The DSM-III criteria also required that the individual who developed PTSD had to have been directly exposed to a traumatic event. The DSMIV criteria (APA 1994) significantly broadened the definition of PTSD to include (a) a "personal experience of an event that involves actual or threatened death or serious injury, or other threat to one's physical integrity" (p. 463) or (b) witnessing a comparable traumatic event or even "learning about unexpected or violent death, serious harm, or threat of death or injury experienced by a family member or a close associate" (p. 462). As a consequence of these modifications, the DSM-IV diagnostic criteria for PTSD have been criticized for failing to discriminate between symptoms of PTSD and normal stress reactions (e.g., learning about the 9/ 11 disaster; Wakefield and Spitzer 2002).

\section{Acute Stress Disorder}

The diagnosis of ASD was first introduced in DSM-IV. It refers to an acute stress reaction that occurs within 1 month following exposure to a traumatic event. Although this diagnosis has been criticized on the grounds that there was little evidence to support its inclusion in the DSM-IV (Bryant and Harvey 2000), it has been useful as a predictor of chronic PTSD (Bryant and Harvey 1997). For example, Harvey and Bryant (1998) found that $60 \%$ of the individuals who met the diagnostic criteria for ASD went on to develop chronic PTSD. The major difference between PTSD and ASD is that the latter requires the presence of significant dissociative symptoms, including a subjective sense of numbing, emotional detachment or absence of emotional responsiveness, a reduction in awareness of one's surroundings, derealization, depersonalization, or dissociative amnesia.

Bryant and Harvey (1997) have stressed that the role of dissociation in ASD is complicated by the ambiguity concerning when the dissociative response occurs. They point out that, according to the DSM-IV criteria, the dissociative symptoms may occur during or after experiencing the traumatic event. This time frame, however, stands in contrast to the requirement that the intrusive avoidance and arousal symptoms need to be experienced as ongoing problems. This also contrasts with the DSM-IV criteria that the symptoms of ASD persist for a minimum of 2 days following the traumatic experience.

The DSM-IV diagnostic criteria for ASD also place more stringent requirements on intrusive, avoidance, and arousal symptoms than PTSD. Although numerous structured interviews and self-report inventories currently exist to assess PTSD, only a few inventories have been developed to assess ASD (Briere 2001; Bryant et al. 2000; Bryant and Harvey 2000).

\section{Posttraumatic Stress Disorder}

PTSD refers to the development of a set of specific psychiatric symptoms following an individual's exposure to terrifying events, such as combat, fire, flood, molestation, natural disasters, sexual assault, actual or threatened death or serious injury, or witnessing someone become badly injured or 
killed. According to the revised fourth edition of the DSMIV-TR (American Psychiatric Association 1994), an individual who develops PTSD must be "confronted with an event or events that involved actual or threatened death or serious injury, or threat to the physical integrity of self or others" which produces intense feelings of "fear, helplessness or horror" (p. 467).

The diagnostic criteria for PTSD further require that the traumatic event be persistently re-experienced by symptoms such as recurrent and intrusive memories, nightmares, a sense of re-living the trauma, psychological and physiological distress when reminded of aspects of the trauma, recurrent or intrusive recollections, distressing dreams, and flashbacks, or by stimuli which symbolize or resembles some aspect of the traumatic event; conscious efforts to avoid specific thoughts, feelings, people, places, or activities which could trigger recollections of the event; and symptoms of emotional arousal (e.g., sleep disturbance, irritability, difficulty concentrating, hypervigilance) and heightened reactivity (e.g., exaggerated startle responses).

While individuals with PTSD typically report symptoms such as nightmares, startle responses, avoidance, flashbacks, and intrusive recollections of the traumatic event, many of these individuals will also complain of symptoms such as impaired concentration, memory, learning, school and work performance, and decision-making skills; confusion, social withdrawal, problems with intimacy, diminished libido, reduced insight, rigid thinking, exhaustion, headaches, insomnia, irritability, anger, poor frustration tolerance, sensitivity to noise and lights, depression, and cardiovascular, gastrointestinal, and musculoskeletal disorders (Kennedy et al. 2007; McMillan et al. 2003).

\section{Traumatic Brain Injury}

The outcome from a TBI varies from death, vegetative state, and severe cognitive and behavioral limitations to a full recovery. The Glasgow Coma Scale (GCS) is the most frequently used measure to determine the severity of a brain injury (Teasdale and Jennett 1974). It is based on a patient's motor, verbal, and eye-opening responses following a TBI and is commonly used by paramedics at the scene of the accident and physicians in emergency departments. A patient's GCS score can range from 3 to 15 . GCS scores that range from 13 to 15 are indicative of a mild brain injury; scores from 9 to 12 are indicative of a moderate brain injury; and scores ranging from 3 to 8 are indicative of a severe brain injury (Rimel et al. 1981). It is important to recognize that individuals who are unable to understand or communicate in English, are deaf, are intubated, are in severe pain, or are under the influence of alcohol, drugs, and/or pain medications at the time they are examined can have spuriously low GCS scores (Sbordone et al. 2007).
A TBI can be caused by a direct physical blow, a penetrating injury (such as a bullet), or mechanical forces that result in rapid acceleration-deceleration of the head. These acute or primary injuries can include brain lacerations, intracranial hematoma, or coup/contrecoup contusions to the brain. Secondary damage to the brain in form of metabolic changes can result in brain swelling, elevated intracranial pressure, cerebral hypoxia, arterial hypoxemia, hydrocephalus, intracranial infections, posttraumatic epilepsy, or anemia (Jennett and Teasdale 1981). In general, the more severe the acute brain injury, the greater the likelihood that a patient will develop debilitating secondary brain damage.

Irrespective of the site of head impact, neuroimaging studies of individuals with TBI have shown that the prefrontal and anterior temporal lobes of the brain are the most likely areas of the brain to sustain damage as a result of the accelerationdeceleration of the brain within the skull (Kolb and Whishaw 2003). Individuals who sustain extensive prefrontal lobe damage might exhibit the following symptoms: dramatic change in their personality; inability to regulate their behavior and emotions; a loss of initiative, curiosity, exploratory behavior, motivation, creativity, and sex drive; difficulty organizing their thoughts, plan, remain on task, monitor their actions, solve problems, recognize their mistakes or rectify them when they are pointed out; impaired social behavior, inflexible thinking, poor judgment, impulsivity, egocentricity, apathy, difficulty showing affection or feeling compassion toward others, use of crude or coarse language in inappropriate circumstances; lack of plans or concern for future events, an inability to profit from experience, perseverative behavior, and a tendency to confabulate when asked to recall information (Stuss and Benson 1986). Individuals who sustain damage to the anterior temporal lobes frequently exhibit the following symptoms: anger outbursts following relatively mild provocation, memory difficulties for recent events, psychiatric disturbances such as depression, and hallucinations, rapid mood swings, and seizures (Saul 1993).

Bullets fired from rifles or handguns at high velocities can penetrate the skull and produce extensive tissue damage within the brain. Bullets that penetrate the lower levels of the brain stem usually produce spontaneous death. Although the mortality rate of missile injuries to the brain is approximately $88 \%$, patients who do not die typically exhibit the following symptoms: significant cognitive impairments, difficulties with recent memory, slow thinking, indifference, concentration difficulties, poor judgment, emotional lability, rapid fatigue, focal neurological deficits, and focal seizures (Adams et al. 1997).

\section{Mild Traumatic Brain Injury}

Although over $80 \%$ of all TBIs are of mild severity, the diagnosis of mild traumatic brain injury (MTBI) is less 
definitive and more diagnostically challenging than more severe TBI. Prior to identifying the diagnostic challenges, we will first provide a definition of MTBI. The Mild Traumatic Brain Injury Committee of the Head Injury Interdisciplinary Special Interest Group of the American Congress of Rehabilitation Medicine (1993) defined MTBI as an injury to the brain caused by acceleration-deceleration forces with or without physical trauma to the head that produces physiological disruption of brain function manifested by at least one of the following criteria: (1) any loss of memory for events immediately before or after the accident; (2) any alteration in mental state at the time of the accident (e.g., feeling dazed, disoriented, or confused); (3) focal neurological deficits that may or may not be transient; (4) and a loss of consciousness of approximately $30 \mathrm{~min}$ or less, an initial GCS score of 13 to 15 , and posttraumatic amnesia (PTA) of $<24 \mathrm{~h}$.

The Collaborating Centre Task Force on Mild Traumatic Brain Injury of the World Health Organization (Carroll et al. 2004) comprehensively examined research studies on MTBI and recommended the continued application of the four diagnostic criteria outlined above. For a detailed description of clinically applying these criteria in the diagnosis of MTBI, see a published educational paper authored by the Policy and Planning Committee of the National Academy of Neuropsychology (Ruff et al. 2009).

\section{Diagnosing a Gap in Memory}

A key diagnostic criterion for diagnosing MTBI is the patient's perceived gap in memory that includes a loss of memory for events immediately before (retrograde amnesia) and/or after the accident (anterograde amnesia or PTA). This memory disruption, as a rule, is caused by the impact and/or an acceleration-deceleration of the brain. Russell and Smith (1961) defined PTA as the period of time before an individual's memory for ongoing events becomes continuous. When a patient becomes capable of recalling ongoing events in chronological order, the patient's PTA has ended.

Determining the duration of the memory gap can be challenging for several reasons. For example, it is often difficult to determine if the amnesia reported by the patient is caused by TBI or is a reaction to the stressful events that followed, such as being extricated from a running car that could explode or a fear of dying while being transported in an ambulance. Reliably determining the duration of the memory gap is particularly difficult when there are isolated "islands" of memory or brief periods of apparently normal encoding and retrieval (Forrester et al. 1994). In general, the longer the duration of PTA, the more severe the initial TBI and the greater the likelihood of prolonged disability (Sbordone et al. 2007).

\section{Diagnosing Loss of Consciousness}

When individuals have been involved in an MVA, the determination of whether they sustained a loss of consciousness as a result of an MTBI is often based on the assessment of the paramedics and/or observers who were present at the scene of the accident. Without such testimony, the determination of whether a patient was actually unconscious is usually based on the patient's self-report of a loss of consciousness. A patient's self-report can be unreliable because the loss of consciousness can overlap with the PTA. Some patients confuse the duration of their loss of consciousness with the duration of their PTA or dissociative amnesia. For example, some individuals assume that, if they are unable to recall the events that occurred following an accident or injury, they must have been unconscious.

A careful examination of the patient's medical records can be helpful if the records are based on direct observations of paramedics or witnesses. Medical records that simply note the patient's answer to the question of "Where you unconscious?" can be unreliable (Ruff et al. 2009). As an example, some patients may deny any loss of consciousness during a MVA, but are observed by witnesses to be unconscious at the scene of the accident.

\section{Overlap Between the MTBI and PTSD Criteria}

\section{Overlap Between Gap in Memory}

Individuals who are acutely exposed to an emotionally traumatic event often report feeling overwhelmed, dazed, stunned, or confused. In extreme circumstances, they may even experience dissociative symptoms during or after the event that may alter their recollection of the event. If these individuals are unable to recall the event, they may be seen as meeting the MTBI criteria. To avoid misdiagnosing an ASD with an MTBI, Malec (1999) has stressed that health care professionals who examine these individuals should determine whether the change in their mental state is due to an MTBI rather than the individual's physical injuries because many individuals report feeling "stunned" after witnessing a traumatic event or sustaining injuries to parts of their body other than to their head.

The MTBI criteria do not have any clearly defined lower limits. As a consequence, individuals who report a very brief (1-2 s) alteration or loss of consciousness are likely to satisfy the MTBI criteria (Kirby and Long 1997), even though the reported alteration or loss of consciousness cannot be objectively verified by witnesses. Thus, the shorter the duration of loss of consciousness or altered mental state, the more difficult the distinction becomes between a brain-based versus emotionally based gap in the individual's recollection. 


\section{Overlap Between Postconcussion Syndrome and PTSD}

The postconcussion syndrome (PCS) typically refers to persistent cognitive, emotional, and physical symptoms that individuals frequently report after sustaining an MTBI. We will use the term PCS to describe the symptom presentation of individuals who sustain MTBI injuries rather than individuals who sustain more severe brain injuries. PCS commonly includes headaches, sensitivity to light and sound, attention and concentration difficulties, memory deficits, fatigue, dizziness, slower processing speed, and irritability (Bohnen and Jolles 1992).

One of the challenges in distinguishing between the symptoms caused by PTSD and those caused by PCS is that both disorders share similar symptoms. For example, PTSD has been reported to produce symptoms such as confusion, impaired learning, forgetfulness, attention and concentration difficulties, slower processing speed, feeling overwhelmed by once simpler tasks, personality change, impulsivity, reduced insight, rigid thinking, reduced motivation, social withdrawal, reduced relational intimacy, impaired work and school performance, fatigue, insomnia, headaches, and hyperarousal (Kennedy et al. 2007). As a consequence, individuals with PTSD are prone to be misdiagnosed with an MTBI, especially if the diagnosis is based on these persistent residuals rather than the primary injury characteristics that define a concussion (Hickling et al. 1998).

Although MTBI can be diagnosed on the basis of focal neurological signs such a posttraumatic seizures, anosmia, and positive neuroimaging (Ruff et al. 2009), a distinction should be made between verifiable neurological signs and the patient's self-reported symptoms. For example, many health care professionals assume that complaints such as memory and concentration difficulties, headache, dizziness, tinnitus, and hypersensitivity to light and noise are specific diagnostic criteria for MTBI. As a consequence, individuals with ASD or PTSD are often erroneously diagnosed with an MTBI when they are seen in emergency rooms if they have been involved in an MVA or later by their physicians even though they never sustained any trauma to their head or experienced an alteration or loss of consciousness. It should be recognized that these subjective complaints are not specific to a concussion or TBI because they are commonly reported by individuals who never sustained TBI (Binder 1997). For example, approximately $50 \%$ of the individuals who are involved in litigation and have no history of head trauma or brain injury will report similar symptoms, which most likely reflect the stress of litigation (Lees-Haley and Brown 1993). Patients who sustain orthopedic injuries or have psychiatric disorders, such as major depression, or generalized anxiety or panic disorders will frequently complain of these or similar symptoms (Binder 1997). Patients with a variety of disparate medical conditions, such as chronic fatigue syndrome (Wearden and Appleby 1996), gastrointestinal disorders (Hochstrasser and Angst 1996), Graves's disease (Stern et al. 1996), and the common cold (Gunstad and Suhr 2002), will also complain of similar symptoms without any history of head injury. Finally, approximately one third of relatively healthy college students also complain of these symptoms (which are even corroborated by their significant others), even when none of them had a history of MTBI or neurological disease (Gouvier et al. 1988).

Questions About the Coexistence of PTSD and TBI

\section{Does a TBI Prevent the Development of PTSD?}

Since PTSD was first included in DSM-III in 1980, a controversy has emerged in the literature whether an individual who has suffered an accident-related TBI can also develop PTSD. One school of thought (e.g., Sbordone and Liter 1995) has argued that, if the TBI results in a loss of consciousness, the individual would be unable to experience the traumatic event in order to form vivid memories that are necessary to generate intrusive recollections of the traumatic event that are essential for the development of PTSD. An inability to experience the traumatic event as a result of unconsciousness would not satisfy the first DSM-IV-TR diagnostic criterion for PTSD, which specifies that that the person "experienced, witnessed, or was confronted with" the traumatic event, or the second criteria, which states "the person's response (to the traumatic event) involved intense fear, helpless, or horror."

Sbordone and Liter (1995) clinically interviewed 70 patients who had been previously diagnosed with either MTBI or PTSD. Although all of the patients in the PTSD group reported symptoms such as nightmares, flashbacks, intrusive recollections, hypervigilance, and phobic or startle reactions and became emotionally upset whenever they were exposed to stimuli associated with the traumatic event or when they were asked to discuss it, none of the MTBI patients in this study reported having these symptoms. These investigators also found that both MTBI and PTSD patients complained of symptoms such as memory, wordfinding and problem-solving difficulties, distractibility, photophobia, fatigue, diminished libido, and interpersonal difficulties.

Based on these findings, they argued that TBI would prevent the development of PTSD because the individual would not experience the traumatic event if they had been unconscious or had no memory of the event. They also argued that, if the traumatic event occurred prior to the onset of retrograde amnesia caused by TBI (e.g., becoming terrified while riding as a passenger in an automobile that was being driven in a reckless manner for several minutes 
prior to colliding with another vehicle) or after the cessation of anterograde amnesia (e.g., witnessing the death of a family member who sustained massive injuries after regaining consciousness following an MVA), a MTBI patient could develop PTSD as a result of being consciously exposed to subsequent or prior traumatic events.

Their argument received support from several investigators. For example, Mayou et al. (1993) reported that none of the 51 patients who had sustained MTBI as a result of an MVA and reported a loss of consciousness for more than 5 min developed PTSD. Similarly, Warden et al. (1997) found that none of the 47 patients with closed brain injuries they examined developed PTSD if they were unable to recall the traumatic event. Similarly, Malhi and Bartlett (1998) reported that only five MTBI patients $(2.5 \%)$ out of 196 developed PTSD, and all five of these individuals were able to clearly recall the traumatic event. Middelboe et al. (1992) reported that only one patient (2.0\%) was diagnosed with PTSD out of a total of 51 patients who had been admitted to the hospital after a "minor head injury." Max et al. (1998) examined 50 children ranging in age from 6 to 14 utilizing psychiatric assessments that were repeated, 3, 6, 12 , and 24 months following TBI. They found that only two (4\%) of the children were found to have PTSD on at least one of the follow-up assessments. Because these investigators did not exclude children with a preexisting history of PTSD, the diagnosis of PTSD that was given to these two children could have reflected TBI reactivation of preexisting childhood trauma (Berthier et al. 1998).

There is evidence that being unable to recall a traumatic event may protect an individual from developing PTSD. For example, Gil et al. (2005) administered a questionnaire to determine which patients who sustained a MTBI as a result of an MVA could recall the details of the traumatic accident. They found that, the less able these individuals could recall the traumatic event, the less likely they developed PTSD. In a subsequent study, Bryant et al. (2009) evaluated a total of 459 patients for PTSD symptoms and PTA who had sustained MTBIs and 708 patients who did not sustain a TBI. They found that, when these patients were followed up, 9.4\% of the patients who had sustained an MTBI met the criteria for PTSD in comparison to $11.8 \%$ of the patients who did not sustain a TBI based on PTSD questionnaires and structured PTSD interviews. They concluded that longer PTA appeared to be protective against selective re-experiencing symptoms. Flesher et al. (2001) assessed 70 patients who had been involved in MVAs for PTSD. They found that patients who were amnestic for the traumatic event displayed a significantly lower incidence of PTSD symptoms than patients who were able to recall the event.

PTSD has been reported to develop in patients with more severe TBIs (e.g., Bryant 2001; Bryant and Harvey 2000) as a result of the presence of "islands of memory" for the traumatic experience or classical fear conditioning, despite impaired consciousness. Harvey et al. (2003) reviewed a total of 23 published studies that examined the prevalence of PTSD across mild, moderate, and severe levels of TBI. They reported that the studies fell into two groups: studies that reported that a substantial proportion (14-40\%) of individuals with TBI developed PTSD and those that found very few cases of PTSD in TBI patients. They noted that studies that reported PTSD after TBI utilized PTSD questionnaires and tests to determine whether a TBI patient had PTSD, whereas the second group relied primarily on unstructured clinical interviews and did not utilize any PTSD tests or questionnaires to determine whether the TBI patients had PTSD.

These investigators failed to point out that questioning TBI patients with cognitive deficits could result in these patients inaccurately endorsing PTSD symptoms they did not have. Although Bryant (2001) also cites a number of studies which demonstrate that patients with moderate and severe TBI can develop PTSD, a review of these studies reveals that the investigators in the studies relied heavily on PTSD questionnaires and structured PTSD interviews to diagnose PTSD even though these TBI patients most likely had cognitive deficits which had been expected to cause them difficulty understanding the tests and questions they were given. Moreover, McMillan (2001) has pointed out that the use of structured PTSD interviews and PTSD questionnaires may result in the misdiagnosis of PTSD in individuals who have TBI. He cited the example of a 21year-old male who had sustained a severe TBI that had disrupted his cognitive functioning and ability to return to work. He noted that, when this patient was assessed 5 months post-injury on the Impact of Events Scale (Horowitz et al. 1979), the patient's score on this test was consistent with individuals who have been diagnosed with PTSD and also satisfied the DSM-IV criteria for PTSD. He also noted that, on the Posttraumatic Diagnostic Scale (Foa et al. 1993), this patient's severity score qualified him for a diagnosis of PTSD. Despite this patient being diagnosed with PTSD as a result of his test scores, McMillan found that a detailed clinical interview did not support a diagnosis of PTSD. He pointed out that the patient's responses to the questionnaires were significantly colored by his cognitive deficits and the general disruption to his life that his brain injury had caused. He also noted that many of the PTSD symptoms this patient endorsed were commonly reported symptoms of TBI.

McMillan's study demonstrates that PTSD can be misdiagnosed in patients with severe TBI when PTSD questionnaires are used to diagnose PTSD. He noted that this was likely to occur whenever brain-injured patients are evaluated with PTSD questionnaires because these patients 
may not follow the questionnaire instructions consistently as a result of their cognitive impairments at the time of testing and erroneously endorse PTSD symptoms. He also noted that the patients were likely to have physical disabilities and other injuries that most likely interfered with their day-to-day functioning. McMillan stressed that this case study suggests that even sophisticated PTSD questionnaire measures can be misleading when evaluating TBI cases for the presence of PTSD.

In subsequent studies, Sumter and McMillan (2005, 2006) administered PTSD tests and questionnaires to 34 patients who had sustained severe traumatic brain injuries to determine whether they also had PTSD. They found that the majority of these patients $(59 \%)$, based on their responses on the questionnaires, met the diagnostic criteria for PTSD. However, when a structured clinical interview was used to diagnosis PTSD, only one (2.9\%) of the 34 patients actually met the diagnostic criteria for PTSD. These investigators found that many of the TBI patients failed to follow the questionnaire instructions and erroneously endorsed PTSD symptoms because of their cognitive difficulties. Based on these findings, they stressed that reliance on PTSD questionnaires to diagnose PTSD in TBI patients is likely to result in a misdiagnosis of PTSD.

In sum, research suggests that the conflicting findings that have been reported in the literature on the relationship between TBI and PTSD could be, at least in part, due to the different methodologies that have been utilized in these studies. For example, studies that reported the relative absence of PTSD following TBI relied on clinical interviews of TBI patients. Studies that diagnosed PTSD appear to have relied on PTSD questionnaires that were not specifically designed to accurately discriminate between the symptoms of PTSD and TBI.

\section{Can TBI Increase the Risk for PTSD?}

Landre et al. (2006) have argued that TBI can diminish an individual's cognitive resources, which may seriously compromise the individual's ability to cope with psychological trauma. Other investigators have suggested that a TBI may cause an individual to develop inappropriate cognitive strategies following the exposure to trauma, which enhances the likelihood that PTSD will develop (Bryant and Guthrie 2007). Hoge et al. (2008) examined combat troops who had returned from Iraq and Afghanistan and found that soldiers who sustained a TBI, usually as a result of blast concussions, had higher rates of PTSD than soldiers who were not exposed to concussive explosions. These findings, however, have been criticized by Bryant (2008); he stressed that the study did not properly diagnose TBI nor accurately discriminate between symptoms involving impaired awareness caused by acute stress reactions and
TBI. Das (2008) and Xydakis et al. (2008) also criticized this study for failing to provide information on the soldiers' premorbid psychiatric history because previous studies have shown that significantly large numbers of subjects with depression after TBI had a preexisting history of depression.

Koenigs et al. (2008) compared 193 Vietnam War combat veterans who suffered penetrating head injuries with 52 combat veterans without brain injury. These 245 veterans were evaluated for PTSD using the structured clinical interview for PTSD (SCID-N/P; First et al. 1994) for DSM-IV-TR axis I disorders. Computed tomography scans were used to determine the lesion volume and location. They found that the incidence of PTSD was substantially less in veterans who had sustained damage to either the ventromedial prefrontal cortex or the anterior temporal area of the brain that included the amygdala.

In sum, these studies address the synergistic effects among PTSD and TBI symptoms. The study of Koenigs et al. (2008) suggests that, if specific regions of the brain that play an important role in PTSD are damaged, the likelihood of developing PTSD is diminished.

\section{How can TBI Patients Develop PTSD?}

Although PTSD and MTBI can occur within the same traumatic context, it is important to ascertain when and what caused the PTSD and MTBI. For example, King (1997) described a 21-year-old unemployed man who had been struck from behind by an automobile while hitchhiking. Although this patient was given the diagnosis of a "mild head injury" at the time he was admitted to the hospital, he also developed PTSD. A careful review of this case revealed that this patient recalled lying in the road shortly after he was struck by the vehicle. He recalled seeing the car that hit him turn around and head directly at him as he lay on the ground. As a result of his orthopedic injuries, he was unable to transport himself to the side of the road and believed that the driver was intentionally trying to kill him in order to "finish him off." Thus, he was exposed to a terrifying event in which he believed he would be intentionally killed that resulted in his developing PTSD after his anterograde amnesia ended.

Although McMillan (1996) reported on 10 cases (3.2\%) that had developed PTSD symptoms from a total of 312 patients who had sustained TBI ranging in severity from mild to severe, a careful review of these cases reveals that the patients who developed PTSD symptoms were able to either recall being in a state of shock shortly after the accident, being trapped in their vehicle, being in pain or physically injured, or witnessing the death or distress of passengers who had been injured during the accident. Four of these cases complained of survivor guilt as a result of 
witnessing the death of two passengers (a child and an adult passenger), as well as the injuries that the other passengers had sustained. Thus, the development of their PTSD appeared to be based on their witnessing the traumatic events that occurred after their anterograde amnesia ended.

Case Studies Next, we describe three clinical cases that illustrate that an individual with a TBI can develop PTSD as a result of being exposed to different traumatic events prior to or following their TBI. The first case involves a young woman who was sexually assaulted and physically beaten to the point where she was rendered unconscious. Although she reported symptoms consistent with both PTSD and TBI after this incident, the etiology of her PTSD involved being brutally raped and beaten for which she had vivid recall. The etiology of her TBI was likely attributable to the blows she sustained to her head while she was beaten, which caused her to lose consciousness. The second case involves an older woman who was driving an automobile that was following a truck that was carrying a crane that fell onto the hood of her vehicle while she was traveling down the freeway. When the truck driver tried to stop his vehicle, the woman's car was thrust back and forth across various freeway lanes multiple times. Several minutes later, when both her vehicle and the truck came to a stop, the woman's car was rear-ended by a bus. As a result of her fear of dying as her car was being thrust back and forth on the freeway, she developed PTSD. The subsequent impact of the rear-end collision caused a significant blow to her head and a loss of consciousness that resulted in a TBI. In the third case, a middle-aged father was diagnosed with a TBI following an MVA in which his daughter was riding as a passenger in his vehicle. When he regained consciousness in the hospital, he saw that his daughter, who was in the next bed, had been severely injured during the accident. Seeing his severely injured daughter caused him to experience intense feelings of fear, helplessness, and terror, which caused him to develop PTSD. Thus, these cases illustrate how individuals with TBI can develop PTSD as a result of being exposed to additional traumatic events if these events occur outside of their TBI-caused retrograde and anterograde amnesia.

What remains unresolved is the controversy whether an individual who has sustained a loss of consciousness as a result of a TBI can develop PTSD without being exposed to another contiguous traumatic event. Based on our review of this literature, the disparate findings that have been reported in the literature may reflect the methodology that the investigators utilized in these studies. For example, studies that relied on structured PTSD interviews and questionnaires to diagnosis PTSD rarely mention any exposure to any contiguous traumatic events other than the TBI. Studies that relied on more comprehensive clinical interviews of
TBI patients often report that the MTBI patients recalled being exposed to a clearly defined contiguous traumatic event that caused them to develop PTSD after their retrograde and anterograde amnesia ended.

McMillan's (2001) study that examined this issue found that the diagnosis based on the data from PTSD questionnaires and structured PTSD interviews resulted in inaccurate diagnosis of PTSD in a patient with TBI. He found that, when a more comprehensive clinical interview was conducted with the patient, there was no evidence that this patient had PTSD. A review of these studies suggests that PTSD can develop if an individual with TBI is exposed to contiguous traumatic events while they are not experiencing retrograde or anterograde amnesia. Thus, if one relies on the definitions of PTSD and MTBI as respectively set forth in DSM-IV-TR and by the American Congress of Rehabilitation Medicine, then two or more distinct and separate traumatic events or etiologies must occur for the diagnoses of MTBI and PTSD to coexist.

Since PTSD questionnaires or structured interviews typically do not have any built-in scales to detect symptom exaggeration or secondary gain, it is difficult to determine whether a patient's responses on these questionnaires or during the structured clinical interviews are accurate or exaggerated, although Briere has developed one, the "Detailed Assessment of Posttraumatic Stress" (Briere 2001). This leaves open the possibility that a TBI patient's endorsement of PTSD symptoms may be due to pending litigation or secondary gain, which should be considered (Bryant et al. 1998).

Lees-Haley and Dunn (1994) have shown that a vast majority of individuals are able to accurately report PTSD symptoms without having any history of PTSD. For example, they found that $85 \%$ of the individuals with no history of PTSD were able to accurately endorse PTSD symptoms and experiences to satisfy the DSM-IV diagnostic criteria of PTSD. These authors stressed that this mostly likely reflects the fact that lay people may have some basic knowledge of PTSD as a result of exposure to books, magazines, and other media hosted by psychologists and psychiatrists. Thus, an individual's scores on a PTSD questionnaire or structured interview could reflect a motivation for secondary gain.

In sum, a TBI does not, as a rule, cause a PTSD. If PTSD symptoms are part to the patient's presentation, then it is essential that separate etiologies are identified in the clinical interview that account for the causation of PTSD and MTBI.

\section{Do PTSD Patients have Cognitive Deficits?}

Although it has been repeatedly shown that individuals who sustain TBI exhibit cognitive deficits on neuropsychological tests and measures, attorneys often assume that, if a 
patient exhibits cognitive deficits on neuropsychological tests following an MVA, they have a TBI rather than PTSD. A review of the research literature reveals that this assumption is at variance with neuropsychological studies that have evaluated the cognitive functioning of PTSD patients. For example, PTSD patients have been shown to have deficits on neuropsychological tests that assess attention, word retrieval, comprehension, psychomotor speed, concentration (Barrett et al. 1996; Crowell et al. 2002; Leskin and White 2007; Neylan et al. 2004), immediate verbal and visual memory (Bryant 2003; Bremner et al. 1993; Everly and Horton 1989; Uddo et al. 1993), and auditory verbal learning and memory (Uddo et al. 1993; Yehuda et al. 1995). More recently, Tischler et al. (2006) administered the Rey Auditory Verbal Learning Test (RAVLT; Rey 1964; Schmidt 1996) to male combat veterans with and without a history of PTSD. They found the veterans with severe PTSD performed significantly worse on the RAVLT. Yehuda et al. (2007) administered the Wechsler Logical Memory and Digit Span subtests (Wechsler 1997) to veterans with and without PTSD. Although the veterans with PTSD did not differ from veterans without PTSD in hippocampal volume as measured by magnetic resonance imaging scan, they performed significantly worse on the Wechsler Logical Memory and Digit Span subtests. Similarly, Gueze et al. (2008) found that, although veterans with PTSD had similar intelligence quotient test scores to veterans without PTSD who were matched for age, year of deployment, and country of deployment, they performed significantly poorer on the RAVLT. Brenner et al. (2009) found that patients with PTSD tested as poorly as TBI patients on the Paced Auditory Serial Addition Test (Gronwall 1977), RAVLT, and the Conners Continuous Performance Test-II (Conners 1992).

In sum, the above-cited literature has documented that reduced neuropsychological functioning is common in patients with PTSD alone. Individuals with PTSD were found to have cognitive deficits particularly in the areas of memory and learning as well as attention and concentrations. Since these areas are also most often compromised in patients with TBI, further research is warranted to determine if a differentiation is possible.

\section{Are the Cognitive Deficits of PTSD Patients due to PTSD-Induced Brain Changes?}

It is generally well-known that the frontal lobes of the brain play a crucial role in a patient's attention, concentration, executive functions, judgment, and ability to encode and retrieve information from memory (Keane et al. 1987; Oscar-Berman and Bardenhagen 1998). The frontal lobes also play a major role in the regulation of impulses, affect, mood stability, and disinhibition (Fuster 1997; Stuss and Benson 1986). Because these processes are often affected in patients with PTSD, Wolfe (1994) has suggested that patients with PTSD are likely to have frontal system abnormalities. To test this hypothesis, Koenen et al. (2001) administered neuropsychological tests and measures that had been shown by prior research (e.g., Oscar-Berman and Bardenhagen 1998) to be sensitive to frontal lobe dysfunction and standard neuropsychological tests to PTSD patients to identify deficits in function related to the prefrontal brain systems. They found that PTSD patients exhibited deficits on working memory measures (e.g., delayed response and delayed alternation), which have been associated with frontal lesions in both nonhuman primates as well as patients with frontal system pathology (Oscar-Berman and Bardenhagen 1998).

Another brain region that has been suggested to play a critical role in the development of PTSD is the hippocampus. The hippocampus plays a key role in the consolidation of novel memories of facts and events into long-term memory and is very sensitive to the effects of stress (Bremner 2007). For example, animal studies have shown that stress adversely affects the hippocampus by producing increased excitatory amino acids, decreased brain-derived neurotrophic factor, and/or increased glucocorticoid activity. These processes have been associated with a loss of the branching of neurons in the hippocampus and the inhibition of hippocampal neurogenesis (Sapolsky 1996).

Individuals who are exposed to traumatic events and develop PTSD generally perform significantly worse on hippocampal-based declarative memory and learning tasks than individuals without PTSD (Francati et al. 2007). A number of investigators have suggested that the relatively poor performance of PTSD patients on declarative memory tasks could be due to a reduction in the volume of the hippocampus (Bremner 2007). Although a number of neuroimaging studies have reported a significant decrease in the volume of the hippocampus in individuals with PTSD (Bremner 2002, 2006, 2007), some investigators have pointed out that these findings are inconsistent as a result of the heterogeneity among the patients used in these studies, which also included a range of traumatic experiences. For example, Francati et al. (2007) have argued that the inconsistencies in these studies were most likely due to the wide array of patients included in them, particularly with respect to their gender and the various causes of their PTSD (e.g., MVA, sexual assault, combat situations, natural disasters). When these factors were controlled, no differences in hippocampal volume were found in comparison to controls, even when the hippocampal volume was corrected by total brain volume or was correlated with the clinical data in patients who had been exposed to trauma in the past (Jatzko et al. 2006). These investigators, however, noted 
that their negative findings could reflect an insufficient length of time since traumatic exposure because the studies which have reported hippocampal volume reduction were based on post-trauma durations of longer than 18 years.

Woon and Hedges (2008), using a meta-analysis of 21 childhood and adult studies, did not find a reduction in the hippocampal volumes of children with maltreatment-related PTSD. They found, instead, that adults who had been exposed to maltreatment during their childhood had reduced hippocampal volume in comparison to healthy controls. Their findings suggest that the reduction in hippocampal volume might not be apparent until these children become adults. Their findings are also consistent with studies using animal models that have shown a link between early life stress and later hippocampal abnormalities (Brunson et al. 2001; Sanchez et al. 2001).

The process of myelination which begins in early childhood is believed to be crucial for normal adult brain function because it increases neuronal transmission speed and the integration of information across brain regions. Bartzokis et al. (2001) have suggested that exposure to stress resulting in PTSD during childhood could interfere with this process and increase the likelihood of excretory amino acid toxicity of the oligodendrocytes, which play a crucial role in myelinization. This disruption would cause a significant decrease in the speed of information processing and disruptions in the brain's ability to process information. Thus, children who develop PTSD as a result of severe maltreatment, such as frequent physical abuse or torture, could have less myelination and difficulty processing information when they become adults in comparison to adults who did not develop PTSD as a result of childhood maltreatment.

In summary, these studies show that PTSD patients appear to have abnormalities in their frontal brain systems and on tasks involving their declarative memory, which are mediated by the hippocampus. Although a number of studies have shown that PTSD can reduce the volume of the hippocampus, recent studies have shown that PTSD in childhood is not likely to reduce the volume of the hippocampus until these children reach adulthood. PTSD during childhood may also interfere with the process of myelination in the brain, which is crucial for normal brain function since it increases neuronal transmission speed, and the integration of information from different regions of the brain.

\section{Can Clinical Interviews of PTSD Patients and/or the Administration of PTSD Questionnaires Negatively Affect their Performance on Neuropsychological Tests?}

Clinical interviews and PTSD questionnaires can trigger terrifying memories and cause PTSD patients to painfully relive the traumatic event. As a consequence, PTSD patients may remain emotional and frightened after the clinical interview and/or the questionnaire has been completed. Unfortunately, their negative emotional state may compromise their performance on the neuropsychological tests they are subsequently administered that day (Sbordone and Purisch 1996; Tischler et al. 2006). To avoid this negative effect on testing, the examiner should consider delaying an in-depth exploration of PTSD until the neurocognitive testing has been completed.

\section{Summary and Conclusions}

There has been a great deal of controversy about the coexistence of TBI and PTSD, particularly when individuals who sustain a TBI are unconscious and/or have amnesia. Some investigators have argued that these individuals are unable to develop PTSD because they are unable to consciously experience feelings of fear, helplessness, and horror as a result of not being able to directly witness or experience a traumatic event. Other investigators have reported that individuals who sustain TBI ranging from mild to severe and were rendered unconscious for periods of time can develop PTSD. A careful review of the methodologies employed in these studies reveals that investigators who relied on clinical interviews of patients found little or no evidence of PTSD in TBI patients. Investigators who relied on structured PTSD interviews and questionnaires found considerable evidence of PTSD in TBI patients.

Studies that examined the efficacy of the different methodologies utilized in these studies to accurately diagnose PTSD found that TBI patients often failed to follow the questionnaire instructions and erroneously endorsed PTSD symptoms as a result of their cognitive difficulties. Moreover, the tests are typically not designed to distinguish between TBI and PTSD symptoms. As a consequence, studies that have relied on PTSD questionnaires to determine whether a patient has developed PTSD after sustaining TBI are at risk of inaccurately diagnosing PTSD.

Based on our review of TBI and PTSD literature, we found no clear evidence that a TBI alone will produce PTSD. Instead, we found that the development of PTSD depends on the individual's exposure to other traumatic events (e.g., being trapped in a motor vehicle that is believed to be leaking gasoline; witnessing serious injuries or the death of a passenger; fearing dying as a result of excessive bleeding after sustaining severe physical injuries) that occur outside the retrograde and anterograde amnesia caused by TBI. Although it seems apparent that the use of PTSD tests and questionnaires might result in the misdiag- 
nosis of PTSD in patients with severe TBI, further research needs to be done to determine the degree to which this misdiagnosis occurs in patients who have milder forms of TBI.

Because PTSD questionnaires or structured interviews typically do not have built-in scales to detect symptom validity, it is difficult to determine whether a patient's responses on these questionnaires or during an interview are accurate or exaggerated. This leaves open the possibility that a TBI patient's endorsement of PTSD symptoms could be due to pending litigation or secondary gain.

Although health care professionals and attorneys use neuropsychological testing to reveal cognitive deficits in TBI patients, they may mistakenly assume that patients who exhibit cognitive difficulties have a TBI rather than PTSD. In this regard, a review of the research literature has shown that PTSD patients with no history of TBI can also have cognitive deficits as a result of their PTSD when they are administered neuropsychological tests.

PTSD patients appear to have abnormalities in their frontal brain systems and on tasks involving their declarative memory that are mediated by the hippocampus. Although a number of studies have shown the PTSD can reduce the volume of the hippocampus, recent studies have shown that PTSD in childhood is not likely to reduce the volume of the hippocampus until these children become adults. PTSD during childhood may also interfere with the process of myelination in the brain, which is crucial for normal brain function because it increases neuronal transmission speed and the integration of information from different regions of the brain.

Clinical interviews and PTSD questionnaires may trigger terrifying memories of the traumatic event and cause PTSD patients to painfully relive them. Unfortunately, their negative emotional state may compromise their performance on neuropsychological tests they are subsequently administered that day.

Overall, then, neuropsychologists must tread carefully in diagnosing PTSD concurrently with TBI. They should interview the patient in a comprehensive manner and ascertain to what time frame putative PTSD-related memories refer to. Also, they should administer the best available self-report and neuropsychological instruments to clarify the emotional and cognitive deficits involved. Finally, they should be aware of litigation factors, such as reasons for exaggerating symptoms, secondary gain, and malingering. Attorneys who engage neuropsychologists on complex cases such as these should rely on the expertise of the neuropsychologists instead of aiming for facile explanations of the disorders and impairments found in the assessments.

The paper illustrates that workers in the area of psychological injury and law face the most difficult diagnostic decisions. The complicating and confounding effects of comorbidities, such as the one illustrated in this paper (of PTSD and TBI), are not always appreciated in assessments in the area and in court. Moreover, we have not addressed even more complex scenarios, such as patients with TBI, PTSD, chronic pain, and sleep disruption. Assessors must make every effort to disentangle these complex cases, while attending to response biases, as described, so that they can defend their methods and testimony in court.

Acknowledgements The authors wish to thank John Briere, Ph.D. for his generous assistance and suggestions.

Open Access This article is distributed under the terms of the Creative Commons Attribution Noncommercial License which permits any noncommercial use, distribution, and reproduction in any medium, provided the original author(s) and source are credited.

\section{References}

Adams, R. D., Victor, M., \& Ropper, A. H. (1997). Principles of neurology (6th ed.). New York: McGraw-Hill.

American Psychiatric Association. (1980). Diagnostic and statistical manual of mental disorders (3rd ed.). Washington: APA.

American Psychiatric Association. (1994). Diagnostic and statistical mental disorders (4th ed.). Washington: APA.

Barrett, D. H., Green, M. L., Morris, R., Giles, W. H., \& Croft, J. B. (1996). Cognitive functioning and post-traumatic stress disorder. American Journal of Psychiatry, 153(11), 1492-1494.

Bartzokis, G., Beckson, M., Lu, P. H., Nuechterlein, K., Edwards, N., \& Mintz, J. (2001). Age related changes in frontal and temporal lobe volumes in men: A magnetic resonance imaging study. Archives of General Psychiatry, 58, 461-465.

Berthier, M. L., Kulisevsky, J., Fernandez-Benitz, J. A., \& Gironell, A. (1998). Reactivation of posttraumatic stress disorder after minor head injury. Depression and Anxiety, 8, 43-47.

Binder, L. R. (1997). A review of mild head trauma, part II: Clinical implications. Journal of Clinical and Experimental Neuropsychology, 19, 432-457.

Bohnen, N., \& Jolles, J. (1992). Neurobehavioral aspects of postconcussive symptoms after mild head injury. Journal of Nervous and Mental Disease, 180, 183-192.

Bremner, J. D. (2002). Neuroimaging studies in post-traumatic stress disorder. Current Psychiatry Reports, 4, 254-263.

Bremner, J. D. (2006). Stress and brain atrophy. CNS and Neurological Disorders-Drug Targets, 5, 503-512.

Bremner, J. D. (2007). Neuroimaging in posttraumatic stress disorder and other stress-related disorders. Neuroimaging Clinics of North America, 17(4), 523-544.

Bremner, J. D., Southwick, S. M., \& Johnson, D. R. (1993). Childhood physical abuse and combat-related post-traumatic stress disorder. American Journal of Psychiatry, 150, 234-239.

Brenner, L. A., Ladley-O'Brien, S. E., Harwood, J. E. F., Filley, C. M., Kelly, J. P., Homaifar, B. Y., et al. (2009). An exploratory study of neuroimaging, neurologic, and neuropsychological findings in veterans with traumatic brain injury and/or posttraumatic stress disorder. Military Medicine, 174, 347-352.

Briere, J. (2001). Detailed assessment of posttraumatic stress. Odessa: PAR. 
Brunson, K. L., Avishai-Eliner, S., Hatalski, C. G., \& Baram, T. Z. (2001). Neurobiology of the stress response early in life: Evolution of a concept and the role of corticotrophin releasing hormone. Molecular Psychiatry, 6, 647-656.

Bryant, R. A. (2001). Posttraumatic stress disorder: Can they coexist? Clinical Psychology Review, 21(6), 931-948.

Bryant, R. A. (2003). Early predictors of posttraumatic stress disorder. Biological Psychiatry, 53, 789-795.

Bryant, R. A. (2008). Disentangling mild traumatic brain injury and stress reactions. New England Journal of Medicine, 385(5), 525526.

Bryant, R. A., \& Guthrie, R. M. (2007). Maladaptive self-appraisals before trauma predict post-traumatic stress disorder. Journal of Consulting and Clinical Psychology, 75, 812-815.

Bryant, R. A., \& Harvey, A. G. (1997). Acute stress disorder: A critical review of diagnostic issues. Clinical Psychology Review, 17, 753-757.

Bryant, R. A., \& Harvey, A. G. (1998). Relationship between acute stress disorder and posttraumatic stress disorder following mild traumatic brain injury. American Journal of Psychiatry, 155, 625-629.

Bryant, R. A., \& Harvey, A. G. (2000). Acute stress disorder: A handbook of theory, assessment, and treatment. Washington: American Psychological Association.

Bryant, R. A., Harvey, A. G., Dang, S. T., \& Sakerville, T. (1998). Assessing acute stress disorder: Psychometric prospective of a structured clinical interview. Psychological Assessment, 10, 215-220.

Bryant, R. A., Moulds, M. L., \& Guthrie, R. M. (2000). Acute stress disorder scale: A self report measure of acute stress disorder. Psychological Assessment, 12(1), 61-68.

Bryant, R. A., Creamer, M., O’Donnell, M., Silove, D., Clark, C. R., \& McFarlane, A. C. (2009). Post-traumatic amnesia and the nature of post-traumatic stress disorder. Journal of the International Neuropsychological Society, 15(6), 862-867.

Carroll, L. J., Cassidy, J. D., Holm, L., Kraus, J., \& Coronado, V. G. (2004). Methodological issues and research recommendations for mild traumatic brain injury: The WHO Collaborating Centre Task Force on Mild Traumatic Brain Injury. Journal of Rehabilitation Medicine, (Suppl. 43), 113-125.

Conners, C. K. (1992). Conners continuous performance test. Toronto: Multi-Health Systems.

Crowell, T. A., Kieffer, K. M., Siders, C. A., \& Vanderploeg, R. D. (2002). Neuropsychogical findings in combat-related posttraumatic stress disorder. Clinical Neuropsychology, 16(3), 310-321.

Das, R. H. (2008). Mild traumatic brain injury in U.S. soldiers returning from Iraq: Letter to editor. New England Journal of Medicine, 359(20), 2178.

Everly, G., \& Horton, A. (1989). Neuropsychology of posttraumatic stress disorder: A pilot study. Perceptual and Motors Skills, 68, 807-810.

First, M., Spitzer, R., Gibbon, M., \& Williams, J. (1994). Structured clinical interview for DSM-IV axis I disorders-Non-patient edition (SCID-N/P Version 2.0). New York: New York State Psychiatric Institute, Biometrics Research.

Flesher, M. R., Delahanty, D. L., Raimonde, A. J., \& Spoonster, E. (2001). Amnesia, neuroendocrine levels and PTSD in motor vehicle accident victims. Brain Injury, 15(10), 879-889.

Foa, E. B., Riggs, D. S., Dancu, C. V., \& Rothbaum, B. O. (1993). Reliability and validity of brief instrument for assessing posttraumatic stress disorder. Journal of Traumatic Stress, 6, 459-473.

Forrester, G., Encel, J., \& Geffen, A. (1994). Measuring posttraumatic amnesia (PTA): A historical review. Brain Injury, 8, $175-184$.

Fox, R. P. (1972). Post combat adaptation problems. Comprehensive Psychiatry, 13, 435-443.
Francati, V., Vermetten, E., \& Bremner, J. D. (2007). Functional neuroimaging studies in posttraumatic stress disorder: Review of current methods and findings. Depression and Anxiety, 24, 202218.

Fuster, J. M. (1997). The prefrontal cortex (3rd ed.). New York: Lippincott-Raven.

Geuze, E., Vermetten, E., Ruf, M., de Kloet, C. S., \& Westenberg, H. G. M. (2008). Neural correlates of associative learning and memory in veterans with posttraumatic stress disorder. Journal of Psychiatric Research, 42, 659-669.

Gil, S., Caspi, Y., Zilderman Ben-Ari, I., Koren, D., \& Klein, E. (2005). American Journal of Psychiatry, 162, 963-969.

Goldsmith, W., \& Cretekos, C. (1969). Unhappy odysseys: Psychiatric hospitalizations among Vietnam returnees. Archives of General Psychiatry, 20, 78-83.

Gouvier, W. D., Uddo-Crane-Crane, M., \& Brown, L. M. (1988). Base rates of post-concussional symptoms. Archives of Clinical Neuropsychology, 3, 273-278.

Gronwall, D. M. A. (1977). Paced auditory serial addition test: A measure of recovery from concussion. Perceptual and Motor Skills, 44, 367-373.

Gunstad, J., \& Suhr, J. (2002). Perception of illness: Nonspecificity of postconcussion syndrome expectation. Journal of the International Neuropsychological Society, 8, 37-47.

Harvey, A. G., Brewin, C. R., Jones, C., \& Kopelman, M. D. (2003). Coexistence of posttraumatic stress disorder and traumatic brain injury: Towards a resolution of the paradox. Journal of the International Neuropsychological Society, 9, 663-676.

Hickling, E. J., Gillen, R., Blanchard, E. B., Buckley, T., \& Taylor, A. (1998). Traumatic brain injury and post traumatic stress disorder: A preliminary investigation of neuropsychological test results in PTSD secondary to motor vehicle accidents. Brain Injury, 12, 265-274.

Hochstrasser, B., \& Angst, J. (1996). The Zurich study: Epidemiology of gastrointestinal complaints and comorbidity with anxiety and depression. European Archives of Clinical Neuroscience, 246, 261-272.

Hoge, C. W., McGurk, D. E., Thomas, J. L., Cox, A. L., Engel, C. C., \& Castro, C. A. (2008). Mild traumatic brain injury in U.S. soldiers returning from Iraq. New England Journal of Medicine, 358(5), 453-463.

Horowitz, M. J. (1976). Stress response syndrome. New York: Aronson.

Horowitz, M. J., Wilner, W. R., \& Alvarez, W. (1979). Impact of event scales: A measure of subjective stress. Psychosomatic Medicine, $41,209-218$.

Jatzko, A., Rothenhofer, S., Schmitt, A., Gaser, C., Demirakca, T., Weber-Fahr, W., et al. (2006). Hippocampal volume in chronic posttraumatic stress disorder (PTSD): MRI study using two different evaluation methods. Journal of Affective Disorders, 94, $121-126$.

Jennett, B., \& Teasdale, G. (1981). The management of head injuries. Philadelphia: F.A. Davis.

Keane, T. M., Wolfe, J., \& Taylor, K. L. (1987). Posttraumatic stress disorder: Evidence for diagnostic validity and methods of psychological assessment. Journal of Clinical Psychology, 43, 32-43.

Kennedy, J. E., Jaffee, M. S., Leskin, G. A., Stokes, J. W., Leal, F. O., \& Fitzpatrick, P. J. (2007). Posttraumatic stress disorder and posttraumatic stress-like disorder like symptoms and mild traumatic brain injury. Brain Injury, 44(7), 895-920.

Kibby, M. Y., \& Long, C. J. (1997). Effective treatment of minor head injury and understanding its neurological consequences. Applied Neuropsychology, 4, 34-42. 
King, N. (1997). Posttraumatic stress disorder and head injury as a dual diagnosis: "Islands" of memory as a mechanism. Journal of Neurology, Neurosurgery, and Psychiatry, 62, 82-84.

Koenen, K. C., Driver, K. L., Oscar-Berman, M., Wolfe, J., Folsom, S., Huang, M. T., et al. (2001). Measures of prefrontal system dysfunction in posttraumatic stress disorder. Brain and Cognition, 45, 64-78.

Koenigs, M., Huey, E. D., Raymont, V., Cheon, B., Solomon, J., Wassermann, E. M., et al. (2008). Focal brain damage protects against post-traumatic stress disorder in combat veterans. Nature Neuroscience, 11(2), 232-237.

Kolb, B., \& Whishaw, I. Q. (2003). Fundamentals of human neuropsychology (5th ed.). New York: Worth.

Landre, N., Poppe, C. J., Davis, N., Schamaus, B., \& Hobbs, S. E. (2006). Cognitive functioning and post-concussive symptoms in trauma patients with and without mild TBI. Archives of Clinical Neuropsychology, 21, 255-273.

Lees-Haley, P. R., \& Brown, R. S. (1993). Neuropsychological complaint base rates of 170 personal injury claimants. Archives of Clinical Neuropsychology, 8, 203-209.

Lees-Haley, P., \& Dunn, J. T. (1994). The ability of naïve subjects to report symptoms of mild brain injury, post-traumatic stress disorder, major depression, and generalized anxiety disorder. Journal of Clinical Psychology, 50(2), 252-256.

Leskin, L. P., \& White, P. M. (2007). Attentional networks reveal executive function deficits in posttraumatic stress disorder. Neuropsychology, 21(3), 275-284.

Malec, J. F. (1999). Mild traumatic brain injury: Scope of the problem. In N. R. Varney \& R. J. Roberts (Eds.), The evaluation and treatment of mild traumatic brain injury (pp. 15-38). Mahwah: Lawrence Erlbaum.

Malhi, G. S., \& Bartlett, J. R. (1998). Loss of consciousness and posttraumatic stress disorder. British Journal of Psychiatry, 173, 537

Mayou, R., Bryant, B., \& Duthie, R. (1993). Psychiatric consequences of road traffic accidents. British Medical Journal, 307, 647-651.

Max, J. E., Castillo, C. S., Robin, D. A., Lindgren, S. D., Smith, W. L., Sato, Y., et al. (1998). Posttraumatic stress symptomatology after childhood traumatic brain injury. Journal of Nervous and Mental Disease, 186(10), 589-596.

McMillan, T. M. (1996). Post-traumatic stress disorder following minor and severe closed head injury: 10 single cases. Brain Injury, 40, 749-758.

McMillan, T. M. (2001). Errors in diagnosing posttraumatic stress disorders after traumatic brain injury. Brain Injury, 15, 39-46.

McMillan, T. W., Hugh-Williams, W., \& Bryant, R. (2003). Neuropsychological Rehabilitation, 13(1/2), 149-164.

Middleboe, T., Andersen, H. S., Birket-Smith, M., \& Friis, M. L. (1992). Psychiatric sequelae of minor head injury: A prospective followup study. European Psychiatry, 7, 183-189.

Mild Traumatic Brain Injury Committee of the Head Injury Interdisciplinary Special Interest Group of the American Congress of Rehabilitation Medicine. (1993). Definition of mild traumatic brain injury. Journal of Head Trauma Rehabilitation, 8, 86-87.

Neylan, T. C., Lenoci, M., Rothlind, J., Metzler, T. J., Schuff, N., Du, A. T., et al. (2004). Attention, learning, and memory in posttraumatic stress disorder. Journal of Traumatic Stress, 17(1), 4146.

Oscar-Berman, M., \& Bardenhagen, F. (1998). Nonhuman primate models of memory dysfunction in neurodegenerative disease: Contributions from comparative neuropsychology. In A. Troster (Ed.), Memory in neurodegenerative disease (pp. 3-20). New York: Cambridge University Press.
Rey, A. (1964). L'examen clinique en psychologie. Paris: Presses Universitaires de France.

Rimel, R. W., Giordani, B., Barth, J. T., Boll, T. J., \& Jane, J. A. (1981). Disability caused by minor head injury. Neurosurgery, 9 , 221-228.

Ruff, R. M., Iverson, G. L., Barth, J. T., Bush, S. S., \& Broshek, D. K. (2009). Recommendations for diagnosing a mild traumatic brain injury: A national academy of neuropsychology education paper. Archives of Clinical Neuropsychology, 24, 3-10.

Russell, W. R., \& Smith, A. (1961). Post-traumatic amnesia in closed head injury. Archives of Neurology, 5, 4-17.

Sanchez, M. M., Ladd, C. O., \& Plotsky, P. M. (2001). Early adverse experience as a developmental risk factor for later psychopathology: Evidence from rodent and primate models. Developmental Psychopathology, 13, 419-449.

Sapolsky, R. M. (1996). Why stress in bad for your brain. Science, $273,749-750$

Saul, R. E. (1993). Neurobehavioral disorders following brain injury, part I-Neurobehavioral sequelae in early stages of recovery. In J. R. Hanley \& M. Belfus (Eds.), Physical medicine and rehabilitation: State of art reviews (pp. 581-591). Philadelphia: Hanley and Belfus.

Sbordone, R. J., \& Liter, J. C. (1995). Mild traumatic brain injury does not produce post-traumatic stress disorder. Brain Injury, 9, 405412.

Sbordone, R. J., \& Purisch, A. D. (1996). Hazards of blind analysis of neuropsychological test data in assessing cognitive disability: Role of confounding factors. Neurorehabilitation, 7, 15-26.

Sbordone, R. J., Saul, R. E., \& Purisch, A. D. (2007). Neuropsychology for psychologists, health care professionals and attorneys (3rd ed.). Boca Raton: CRC

Schmidt, M. (1996). Rey auditory and verbal learning test. A handbook. Los Angeles: Western Psychological Services.

Stern, R., Robinson, B., Thorner, A., Arruda, J., Prohaska, M., \& Prange, A. (1996). A survey study of neuropsychiatric complaints in patients with Graves's disease. Journal of Neuropsychiatry and Clinical Neurosciences, 8, 181-185.

Stuss, D. T., \& Benson, D. F. (1986). The frontal lobes. New York: Raven.

Sumpter, R. E., \& McMillan, T. M. (2005). Misdiagnosis of post traumatic stress disorder following severe traumatic brain injury. British Journal of Psychiatry, 186, 423-426.

Sumpter, R. E., \& McMillian, T. M. (2006). Errors in self-report of post-traumatic stress disorder after severe brain injury. Brain Injury, 20(1), 93-99.

Teasdale, G., \& Jennett, B. (1974). Assessment of coma and impaired consciousness: A practical scale. Lancet, ii, 81-84.

Tischler, L., Brand, S. R., Stravitsky, K., Labinsky, E., Newmark, R., Grossman, R., et al. (2006). The relationship between hippocampal volume and declarative memory in a population of combat veterans with and without PTSD. Annals of New York Academy of Science, 1071, 405-409.

Uddo, M. J., Vasterling, J. J., Bailey, K., \& Sutker, P. B. (1993). Memory and attention in combat-related post-traumatic stress disorder (PTSD). Journal of Psychopathology and Behavioral Assessment, 15, 43-52.

Wakefield, J. C., \& Spitzer, R. L. (2002). Lowered estimates-But of what? Archives of General Psychiatry, 59, 129-130.

Warden, D. L., Labbate, L. A., Salazar, A. M., Nelson, R., Sheley, E., Staudenmeier, J., et al. (1997). Post-traumatic stress disorder in patients with traumatic brain injury with amnesia for the event. The Journal Neuropsychiatry and Clinical Neurosciences, 9, 18 22.

Wearden, A., \& Appleby, L. (1996). Research on cognitive functioning in patients with chronic fatigue syndrome (CFS): What 
conclusions can we draw? Journal of Psychosomatic Research, 41, 197-211.

Wechsler, D. (1997). Wechsler adult intelligence scale III (WAIS-III). New York: Psychological Corporation.

Wolfe, J. (1994). Applying the neuropsychology of memory disorder. In L. S. Cermak (Ed.), Neuropsychological explorations of memory and cognition: Essays in honor of Nelson Butters (pp. 285-293). New York: Plenum.

Woon, F. U., \& Hedges, D. W. (2008). Hippocampal and amygdala volumes in children and adults with childhood maltreatmentrelated posttraumatic stress disorder: A meta-analysis. Hippocampus, 18, 729-736.
Xydakis, M. S., Robbins, A. S., \& Grant, G. A. (2008). Mild traumatic brain injury in U.S. soldiers returning from Iraq: Letter to editor. New England Journal of Medicine, 359(20), 2177.

Yehuda, R., Keefe, R. S. E., Harvey, P. D., Levengood, R. A., Gerber, D. K., Geni, J., et al. (1995). Learning and memory in combat veterans with posttraumatic stress disorder. American Journal of Psychiatry, 152, 137-139.

Yehuda, R., Golier, J. A., Tischler, L., Harvey, P. D., Newmark, R., Yang, R. K., et al. (2007). Hippocampal volume in aging combat veterans with and without post-traumatic stress disorder: Relation to risk and resilience factors. Journal of Psychiatric Research, $41,435-445$. 